\title{
Incidência de tumores pediátricos no Brasil
}

Childhood cancer incidence in Brazil

\author{
Rejane de Souza Reis', Marceli de Oliveira Santos² e Luiz Claudio Santos Thuler ${ }^{3}$
}

\section{Resumo}

No Brasil, ainda são poucos os trabalhos que abordam temas relacionados à epidemiologia dos tumores pediátricos. As informações sobre incidência do câncer provêm dos Registros de Câncer de Base Populacional (RCBP). Atualmente, há no Brasil 22 RCBP implantados. Destes, 17 contêm informaçóes consolidadas disponíveis para análise. Essas informaçôes foram obtidas a partir de um aplicativo informatizado, desenvolvido pelo Instituto Nacional de Câncer (INCA), para entrada e consolidação dos dados colhidos pelos RCBP, o SisBasepop. Apresentase, neste trabalho, uma análise descritiva das informações disponíveis sobre a incidência dos principais tumores pediátricos no país. Observa-se, em geral, uma predominância dos casos de leucemias, que variam de $15 \%$ a $45 \%$ de todos os tumores pediátricos, seguidos dos linfomas (5\% a $25 \%)$ e tumores do sistema nervoso central $(5 \%$ a $22 \%)$. Conclui-se ressaltando que é viável obter dados de incidência de câncer pediátrico, em larga escala, a partir dos RCBP.

Palavras-chave: Neoplasias; Incidência; Criança; Adolescente; Brasil.

${ }^{1}$ Bióloga, Analista de Programas de Câncer Júnior - INCA.

${ }^{2}$ Estatística Epidemiologista, Analista de Programas de Câncer Sênior - INCA.

${ }^{3}$ Médico Epidemiologista - INCA; Professor Adjunto da Universidade Federal do Estado do Rio de Janeiro (UNIRIO).

Endereço para correspondência: Rejane de Souza Reis. Rua dos Inválidos, 212/3o andar - Centro - Rio de Janeiro - RJ - Brasil - CEP: 20.231-020.

E-mails: rejane@inca.gov.br; msantos@inca.gov.br; lthuler@inca.gov.br 


\section{INTRODUÇÃO}

O câncer pediátrico representa de $0,5 \%$ a $3 \%$ de todos os tumores na maioria das populações. Internacionalmente, os tumores pediátricos mais comuns são as leucemias, os linfomas e os tumores do Sistema Nervoso Central (SNC) ${ }^{1}$. Estes estão definidos pela Classificação Internacional do Câncer na Infância (CICI), com base na sua morfologia ${ }^{2}$. A cada dia, porém, a classificação dos tumores pediátricos ganha mais detalhamento, devido aos avanços nas técnicas laboratoriais baseadas na biologia molecular, imunologia e genética celular.

Enquanto os tumores nos adultos estão, em geral, relacionados à exposição a vários fatores de risco como o tabagismo, estilos de vida, alimentação, ocupação e agentes carcinógenos específicos, a maior parte das causas dos tumores pediátricos ainda é completamente desconhecida. Sabe-se ainda que, do ponto de vista clínico, os tumores pediátricos apresentam menores períodos de latência, em geral crescem rapidamente e são mais invasivos, porém respondem melhor ao tratamento ${ }^{3}$.

As informaçôes sobre a incidência do câncer provêm dos Registros de Câncer de Base Populacional (RCBP), que são centros onde a coleta, o armazenamento e a análise da ocorrência de casos novos de câncer em uma população são realizados de maneira sistemática.

As principais fontes notificadoras (qualquer estabelecimento onde é feito diagnóstico ou tratamento de câncer) dos RCBP incluem hospitais especializados, hospitais gerais, hospitais universitários, hospitais militares, laboratórios de anatomia patológica, serviços de quimioterapia e serviços de radioterapia, sejam eles públicos ou privados. Alguns RCBP contam ainda com informaçōes provenientes de clínicas, institutos médicolegais (necropsia) e asilos. Além disso, são utilizadas as informações do Sistema de Informação sobre Mortalidade (SIM), Autorização de Procedimentos de Alta Complexidade em Oncologia (APAC), Autorização de Internação Hospitalar (AIH) e Sistema de Informação do Câncer da Mulher (SisCam).

De acordo com as recomendaçôes internacionais, seguidas pelos RCBP brasileiros, são considerados casos novos os pacientes com o diagnóstico de câncer confirmado por exame histopatológico, citopatológico, hematológico, exploração cirúrgica, exame por imagem, exame clínico (laboratorial ou não) e necropsia. Além disso, o paciente deve ter residência comprovada na área de cobertura do RCBP. São registrados os tumores de localização primária com comportamento biológico maligno, os tumores in situ, os de localização secundária ou metastáticos e os malignos de localização incerta (se primária ou secundária). São excluídos os tumores de comportamento biológico benigno e aqueles de comportamento incerto (se benignos ou malignos) ${ }^{4}$.

A área de cobertura de um RCBP compreende a delimitação geográfica de onde os técnicos obterão os dados de câncer. Para a maioria dos RCBP, a área de cobertura é o próprio município. As exceções são os RCBP de Belém (Belém e Ananindeua), Cuiabá (Cuiabá e Várzea Grande) e Vitória (Grande Vitória: Cariacica, Guarapari, Serra, Viana, Vila Velha e Vitória).

Os dados coletados pelos RCBP compreendem variáveis de identificação (nome do paciente, nome da mãe, número do prontuário ou número do exame); variáveis demográficas (sexo, cor da pele, data de nascimento, idade na data do diagnóstico, profissão, endereço completo) e variáveis referentes ao tumor (localização primária, morfologia, meio de diagnóstico, extensão, data do diagnóstico, data do óbito e se a causa do óbito foi câncer).

No Brasil, os RCBP utilizam o limite de 18 anos de idade para a definição de câncer pediátrico, conforme recomendação do $\mathrm{INCA}^{5}$. A idade limite considerada para o câncer pediátrico e utilizada em muitos estudos é 14 anos, porém este corte etário é arbitrário ${ }^{6}$. Segundo a Agência Internacional para Pesquisa sobre o Câncer (IARC), há três formas de se apresentar as faixas etárias: por idade sem grupamentos; agrupadas com incremento de cinco anos e, quando possível, grupamentos mantendo em separado os casos de idade menor de um ano?

As informações produzidas pelos RCBP são fundamentais para definir o papel de fatores etiológicos e estabelecer prioridades na prevenção, no planejamento e no gerenciamento dos serviços de saúde.

Há atualmente no Brasil 22 RCBP implantados, sendo quase em sua totalidade nas capitais (20 capitais, Distrito Federal e uma cidade não-capital). Uma vez que não há trabalho publicado que inclua uma análise conjunta das informaçōes dos RCBP brasileiros, justifica-se a realização do presente estudo que tem como objetivo descrever o perfil epidemiológico da incidência dos tumores pediátricos nos 17 RCBP brasileiros que possuem informações consolidadas para pelo menos um ano.

\section{METODOLOGIA}

Para este trabalho, foram utilizadas informações sobre a incidência do câncer pediátrico, disponíveis para o período compreendido entre 1991 e 2001 provenientes de 17 RCBP. Destes, 15 estão localizados em capitais (Aracaju, Belém, Belo Horizonte, Cuiabá, Fortaleza, Goiânia, João Pessoa, Manaus, Natal, Palmas, Porto Alegre, Recife, Salvador, São Paulo e Vitória), um no 
Distrito Federal e um no município de Campinas - SP.

Foi utilizada como base para a codificação dos dados coletados a $2^{a}$ edição da Classificação Internacional de
Doenças para Oncologia (CID-O2)2 . Para a emissão do relatório, foi feita uma conversão direta da CID-O2 para a CICI (Quadro 1).

Quadro 1. Classificação Internacional do Câncer na Infância (1996)

\begin{tabular}{|c|c|c|c|c|c|}
\hline \multirow{2}{*}{ Grupos de Diagnósticos } & \multicolumn{2}{|c|}{ Códigos da CID-02 } & \multirow{2}{*}{ Grupos de Diagnósticos } & \multicolumn{2}{|c|}{ Códigos da CID-02 } \\
\hline & Morfologia & Topografia & & \begin{tabular}{|l|} 
Morfologia \\
\end{tabular} & Topografia \\
\hline \multicolumn{3}{|c|}{ I. Leucemia } & \multicolumn{3}{|c|}{ IX. Sarcomas de Partes Moles } \\
\hline (a) Leucernia linfóide & $9820-9827,9850$ & & \multirow{2}{*}{$\begin{array}{l}\text { (a) Rabdomiossarcoma e sarcoma } \\
\text { embreionário } \\
\text { (b) Fibrossarcoma, neurofibrossarcoma e } \\
\text { outras neoplasias fibromatosas } \\
\end{array}$} & $8900-8920,8991$ & \\
\hline \multirow[t]{2}{*}{ (b) Leucernia nẫo linfocitica aguda } & \begin{tabular}{|l|}
$9840,9841,9861,9864,9866$ \\
$9867,9891,9894,9910$
\end{tabular} & & & $\begin{array}{l}8810,8811,8813-8838,9540- \\
9561\end{array}$ & \\
\hline & & & (c) Sarcoma de Kaposi & 9140 & \\
\hline (c) Leucernia mielóide crônica & 9863,9868 & & \multirow{7}{*}{ (d) Outros sarcomas de partes moles } & $8840-8896,8982,8990,9040-$ & \\
\hline \multirow[t]{2}{*}{ (d) Outras leucernias especificadas } & $\begin{array}{l}9830,9842,9860,9862,9870- \\
9890,9892,9893,9900,9930-\end{array}$ & & & $\begin{array}{l}9044,9120-9134,9150-9170 \\
9251,9581\end{array}$ & \\
\hline & & & & - & C00.0-C63.9, \\
\hline (e) Leucernias nẫo especificadas & $9800-9804$ & & & 0903 & \\
\hline \multicolumn{3}{|c|}{$\begin{array}{c}\text { II. Linfomas e Heoplasias Retículo-Endoteliais } \\
\end{array}$} & & $9231,9240,9363,9364$ & $\begin{array}{l}\mathrm{C} 00.0-\mathrm{C} 39.9 \\
\mathrm{C} 47.0-\mathrm{C} 80.9\end{array}$ \\
\hline \multirow{3}{*}{$\begin{array}{l}\text { (a) Doença de Hodgkin } \\
\text { (b) Linfoma nẫo Hodgkin }\end{array}$} & $9650-9667$ & & & & $\mathrm{C} 00.0-\mathrm{C} 39.9$ \\
\hline & $\begin{array}{l}9591-9595,9670-9686,9690- \\
9714,9723\end{array}$ & & & 9260 & C47.0-C76.8 \\
\hline & $\begin{array}{l}9714,9723 \\
9687 \\
\end{array}$ & & $\begin{array}{l}\text { (e) Sarcomas de partes moles nẵo } \\
\text { especificados }\end{array}$ & $8800-8804$ & $\begin{array}{l}\text { Con.0-C39.9 } \\
\text { C44.0-C80.9 }\end{array}$ \\
\hline \multirow{2}{*}{$\begin{array}{l}\text { (c) Linfoma de Burkitt } \\
\text { (d) Miscelânia de neoplasias linfo-reticulares }\end{array}$} & $9720.9731-9764$ & & \multicolumn{3}{|c|}{ X. Heoplasias de Células Germinativas, Trofoblásticas e Outras Gonadais } \\
\hline & $9720,9731-9704$ & & (a) Tumores de células germinativas & $9060-9102$ & $C 70.0-C 72.9$ \\
\hline (e) Linfornas nẫo especificados & 9590 & & intracranianos e intra-espinhais & $0000-3102$ & C75.1-C75.3 \\
\hline \multicolumn{3}{|c|}{ III. SHC e Miscelânia de Heoplasias Intracranianas e Intra-Espinhais } & \multirow{4}{*}{$\begin{array}{l}\text { (b) Outros tumores de células germinativas } \\
\text { nấo gonadais e tumores de células } \\
\text { germinativas nấo gonadais específicados }\end{array}$} & \multirow{4}{*}{$9060-9102$} & $\begin{array}{l}\mathrm{C} 00.0-\mathrm{C} 55.9 \\
\mathrm{C} 57.0-\mathrm{C} 61.9\end{array}$ \\
\hline (a) Ependimoma & $9383,9390-9394$ & & & & C63.0-C69.9, \\
\hline & 9380 & C72.3 & & & C73.9-C75.0, \\
\hline (D) Astructionta & $9381,9400-9441$ & & & & C75.4-C80.9 \\
\hline (c) Tumores neuroectodérmicos primitivos & $9470-9473$ & & $\begin{array}{l}\text { (c) Tumores de células germinativas } \\
\text { gonadais }\end{array}$ & $9060-9102$ & $\begin{array}{l}\text { C56.9, C62.0- } \\
\text { C62.9 }\end{array}$ \\
\hline (d) Outros Gliomas & 9380 & $\begin{array}{l}\text { C70.0-C72.2 } \\
\text { C72.4-C72.9 }\end{array}$ & & \begin{tabular}{|l|}
$8010-8041,8050-8075,8082$ \\
$8120-8122,8130-8141,8143$
\end{tabular} & \\
\hline & $9382,9384,9442-9460,9481$ & & & {$\left[\begin{array}{l}120-012,0130-611,0145, \\
8155,8190-8201,8210,8211\end{array}\right.$} & \\
\hline $\begin{array}{l}\text { (e) Outras neoplasias intracranianas e intra- } \\
\text { espinhais especificadas }\end{array}$ & \begin{tabular}{|l|}
$8270-8281,8300,9350-9362$ \\
$9480,9505,9530-9539$
\end{tabular} & & (d) Carcinomas gonadais & $\begin{array}{l}8221-8241,8244-8246,8260- \\
8263,8290,8310,8320,8323\end{array}$ & $\begin{array}{l}\mathrm{C} 56.9, \mathrm{C} 62.0- \\
\mathrm{C} 62.9\end{array}$ \\
\hline $\begin{array}{l}\text { (f) Neoplasias intracranianas e intra- } \\
\text { espinhais năo especificadas } \\
\end{array}$ & $8000-8004$ & $\begin{array}{l}\mathrm{C} 70.0-\mathrm{C} 72.9 \\
\mathrm{C} 72.1-\mathrm{C} 75.3 \\
\end{array}$ & & $\begin{array}{l}8430,8440,8480-8490,8504 \\
8510,8550,8560-8573\end{array}$ & \\
\hline $\begin{array}{l}\text { IV. Tumores do Siste } \\
\end{array}$ & ma Hervoso Simpático & & & $8380,8381,8441-8473$ & \\
\hline (a) Neuroblastoma e ganglioneuroblastoma & 9490,9500 & & (e) Outros tumores gonadais malignos nẫo & $8590-8670,9000$ & \\
\hline $\begin{array}{l}\text { (b) Outros tumores do sistema nervoso } \\
\text { simpático }\end{array}$ & $\begin{array}{l}8680,8693-8710,9501-9504 \\
9520-9523\end{array}$ & & especificados & $8000-8004$ & $\begin{array}{l}\mathrm{C} 56.9, \mathrm{C} 62.0- \\
\mathrm{C} 62.9\end{array}$ \\
\hline $\begin{array}{ll} & \text { v. Retino } \\
\end{array}$ & oblastoma & & $\begin{array}{r}\text { Xl. Carcinomas e Outras } \\
\end{array}$ & oplasias Malignas Epiteliais & \\
\hline & $9510-9512$ & & (a) Carcinoma de córtex adrenal & $8370-8375$ & \\
\hline $\begin{array}{ll}\text { VI. Tumor } \\
\end{array}$ & res Renais & & & $\begin{array}{l}8010-8041,8050-8075,8082 \\
8120-8122,8130-8141,8155\end{array}$ & \\
\hline (a) Tumor de Wilms, tumor rabdóide e & 8960,8964 & & & $8190,8200,8201,8211,8230$ & \\
\hline sarcoma de células claras & 8963 & $664.9, \mathrm{C} 80.9$ & (b) Carcinoma de tireóide & $8231,8244-8246,8260-8263$ & C73.9 \\
\hline & \begin{tabular}{|l|}
$8010-8041,8050-8075,8082$ \\
$8120-8122,8130-8141,8143$
\end{tabular} & & & $\begin{array}{l}8290,8310,8320,8323,8430 \\
8440,8480,8481,8500-8573\end{array}$ & \\
\hline & $8155,8190-8201,8210,8211$ & & & $8330-8350$ & \\
\hline (b) Carcinoma renal & $\begin{array}{l}8221-8231,8240,8241,8244- \\
8246,8260-8263,8290,8310 \\
8312,8320,8323,8401,8430 \\
8440,8480-8490,8504,8510 \\
8550,8560-8573\end{array}$ & C64.9 & (c) Carcinoma de nasofaringe & $\begin{array}{l}8010-8041,8050-8075,8082, \\
8120-8122,8130-8141,8155, \\
8190,8200,8201,8211,8230, \\
8231,8244-8246,8260-8263, \\
8290,8310,8320,8323,8430, \\
8440,8480,8481,8504,8510, \\
8550,8560-8573\end{array}$ & C11.0-C119 \\
\hline $\begin{array}{l}\text { (c) Tumores renais malignos nẫo } \\
\text { especificados }\end{array}$ & $8000-8004$ & C64.9 & (d) Melanoma maligno & $8720-8780$ & \\
\hline $\begin{array}{lc}\text { VII. Tumore } \\
\end{array}$ & es Hepáticos & & & $\begin{array}{l}8010-8041,8050-8075,8082 \\
8090-8110,8140,8143,8147\end{array}$ & \\
\hline (a) Hepatoblastoma & 8970 & & & $8190,8200,8240,8246,8247$ & \\
\hline & $\begin{array}{l}8010-8041,8050-8075,8082 \\
8120-8122,8140,8141,8143 \\
8155,8160-8180,8190-8201\end{array}$ & & (e) Carcinoma de pele & $\begin{array}{l}8260,8310,8320,8323,8390- \\
8420,8430,8480,8542,8560 \\
8570-8573,8940\end{array}$ & C44.0-C44.9 \\
\hline (b) Hepatocarcinoma & $\begin{array}{l}8210,8211,8230,8231,8240 \\
8241,8244-8246,8260-8263 \\
8310,8320,8323,8401,8430 \\
8440,8480-8490,8504,8510 \\
8550,8560-8573\end{array}$ & $\mathrm{C} 22.0, \mathrm{C} 22.1$ & $\begin{array}{l}\text { (f) Outros carcinomas e carcinomas nẫo } \\
\text { especificados }\end{array}$ & $\begin{array}{l}8010-8082,8120-8155,8190- \\
8263,8290,8310,8314-8323 \\
8430-8440,8480-8580,8940 \\
8941\end{array}$ & $\begin{array}{l}\mathrm{C} 00.0-\mathrm{C} 10.9 \\
\mathrm{C} 12.9-\mathrm{C} 21.8, \\
\mathrm{C} 23.9-\mathrm{C} 39.9 \\
\mathrm{C} 48.0-\mathrm{C} 48.8 \\
\mathrm{C} 50.0-\mathrm{C} 55.9 \\
\mathrm{C} 57.0-\mathrm{C} 61.9 \\
\mathrm{C} 63.0-\mathrm{C} 63.9\end{array}$ \\
\hline $\begin{array}{l}\text { (c) Tumores hepáticos malignos nẫo } \\
\text { especificados }\end{array}$ & $8000-8004$ & $\mathrm{C} 22.0, \mathrm{C} 22.1$ & & & $\begin{array}{l}\mathrm{C} 63 . \mathrm{U}-\mathrm{C} 63.9 \\
\mathrm{C} 65.9-\mathrm{C} 72.9 \\
\mathrm{C} 75.0-\mathrm{C} 80.9\end{array}$ \\
\hline $\begin{aligned} \text { Vill. Tumores ó } \\
\end{aligned}$ & Sseos Malignos & & XII. Outros Tumores $\mathrm{M}$ & lignos não Especificados & \\
\hline (a) Osteossarcoma & $9180-9200$ & & & $8930,8933,8950,8951,8971-$ & \\
\hline (b) Condrossarcoma & $9220-9230$ & & (a) Outros tumores malignos especificados & $8981,9020,9050-9053,9110$ & \\
\hline (b) Condrossarcoma & $9231-9240$ & $\mathrm{C} 40.0-\mathrm{C} 41.9$ & & 9580 & \\
\hline (c) Sarcoma de Ewing & 9260 & $\begin{array}{l}\text { C40.0-C41.9, } \\
\text { C80.9 }\end{array}$ & & & $\begin{array}{l}\mathrm{C} 00.0-\mathrm{C} 21.8 \\
\mathrm{C} 23.9-\mathrm{C} 39.9 \\
\mathrm{C} 42 . \mathrm{C}\end{array}$ \\
\hline & 9363,9364 & C40.0-C41.9 & & & $\begin{array}{l}\text { C42.2-C55.9, } \\
\text { C57.0-C61.9, }\end{array}$ \\
\hline $\begin{array}{l}\text { (d) Outros tumores ósseos malignos } \\
\text { especificados }\end{array}$ & $8812,9250,9261-9330,9370$ & & especificados & $8000-8004$ & $\begin{array}{l}\text { C63.0-C63.9, } \\
\text { C65.9-C69.9, }\end{array}$ \\
\hline $\begin{array}{l}\text { (e) Tumores ósseos malignos nẫo } \\
\text { especificados }\end{array}$ & $\begin{array}{l}8000-8004,8800,8801,8803, \\
8804\end{array}$ & & & & $\begin{array}{l}\text { C73.9-C75.0, } \\
\text { C75.4-C80.9 }\end{array}$ \\
\hline
\end{tabular}


A variável idade foi categorizada em cinco grupos etários: $<1$ ano, 1-4 anos, 5-9 anos, 10-14 anos e 15-18 anos ${ }^{7}$. Vale ressaltar que o RCBP de São Paulo não considera as crianças menores de um ano isoladamente, sendo nesta pesquisa incluídas no grupo de 1 a 4 anos.

O INCA desenvolveu um aplicativo para a informatização dos dados dos RCBP, o SisBasepop. Dos RCBP incluídos neste trabalho, 13 utilizaram este aplicativo desde o processo de entrada de dados até a seleção dos casos elegíveis (Aracaju, Belém, Belo Horizonte, Cuiabá, Fortaleza, João Pessoa, Manaus, Natal, Palmas, Porto Alegre, Recife, Salvador e Vitória); o Distrito Federal utilizou o SisBasepop somente para entrada de dados. Os três RCBP restantes (Campinas, Goiânia e São Paulo) utilizaram sistemas próprios para a informatização dos dados. Estes últimos enviaram as informações já convertidas para a CICI em formato previamente estabelecido.

O SisBasepop verifica a consistência das informações desde a entrada de dados até a escolha do caso. Tais verificaçôes consistem na checagem da compatibilidade de algumas variáveis, tais como: topografia e sexo, topografia e morfologia, morfologia e extensão da doença, data de nascimento e data do diagnóstico, morfologia e idade. Para todos os 17 RCBP, foram emitidos relatórios que continham informaçôes sobre o número de casos novos de tumores pediátricos, por idade e sexo. Foi também gerado um relatório para todos os tumores (pediátricos e adultos) a fim de identificar o número total de casos novos, por período, e definir sua magnitude. Para isso, tabelas comparativas foram elaboradas, por RCBP, mostrando os percentuais dos tumores pediátricos, dos tumores não-pediátricos e o total de tumores, e a relação entre a frequiência relativa das leucemias, dos linfomas, dos tumores do SNC e de outros tumores. Estes últimos incluem os tumores do Sistema Nervoso Simpático, retinoblastoma, tumores renais, tumores hepáticos, tumores ósseos malignos, sarcoma de partes moles, neoplasias de células germinativas, trofoblásticas e outras gonadais, carcinomas e outras neoplasias malignas epiteliais, e outros tumores malignos não-especificados.

Além disso, foram elaborados gráficos comparativos, para os três tumores descritos na literatura como os mais freqüentes (leucemias, linfomas e tumores do $\mathrm{SNC}$ ), a partir das taxas ajustadas por idade. Um diagrama de caixa (box-plot) foi feito com o objetivo de comparar as taxas específicas de incidência por idade e por sexo, para cada RCBP.

Para a elaboração destes gráficos, foram considerados somente os RCBP com dois ou mais anos de informações consolidadas (Belém, Campinas, Distrito Federal, Goiânia, João Pessoa, Natal, Porto Alegre, Recife, Salvador e São Paulo), visto que as informaçōes referentes somente a um ano podem não refletir a incidência real, com a inclusão de casos prevalentes (superestimação) ou a não-cobertura da totalidade das fontes notificadoras (subestimação). A descrição detalhada dos termos estatísticos utilizados encontra-se a seguir.

\section{Distribuição Percentual}

Corresponde à freqüência relativa do número de casos novos dos tumores pediátricos em relação ao total de casos.

Distribuição Percentual $=\frac{\text { número total de casos de tumores infantis }}{\text { número total de casos de tumores }} \times 100$

\section{Taxa Média Específica de Incidência}

Esta taxa refere-se ao risco de ocorrência de casos novos de um evento devido a um determinado atributo como idade e sexo, calculada por meio da seguinte fórmula:

Taxa média de incidência específica $=\frac{\sum_{\text {por faixa etária e sexo no período determinado }}^{\text {número de casos novos de um evento, }}}{\sum_{\text {por faixa etária e sexo no período determinado }}^{\text {populão de refercia em risco, }}} \times 100.000$

A taxa específica de incidência foi calculada por 100.000 habitantes, utilizando-se a população fornecida pelo Instituto Brasileiro de Geografia e Estatística (IBGE) e refere-se à contagem populacional de 1996.

\section{Taxa Média de Incidência Ajustada por Idade}

A utilização da taxa média de incidência ajustada por idade permite minimizar o efeito de diferenças etárias entre populações, de modo que as diferenças encontradas não possam ser atribuídas a diferenças na estrutura etária. Seu cálculo é feito pela fórmula:

Taxa média de incidência ajustada por idade $=\frac{\sum \begin{array}{c}\text { (taxa média de incidência específica por idade } \mathrm{x} \\ \text { população padrão mundial na faixa etária) }\end{array}}{\sum \text { população padrão mundial }}$

A população padrão mundial infantil utilizada foi aquela proposta por Segi (1960), modificada por Doll et al. (1966), conforme apresentada abaixo:

\begin{tabular}{c|c|c}
\hline Índice & Faixa etária & Total \\
\hline 1 & 0 & 2.400 \\
\hline 2 & $01-04$ & 9.600 \\
\hline 3 & $05-09$ & 10.000 \\
\hline 4 & $10-14$ & 9.000 \\
\hline 5 & $15-18$ & 7.200 \\
\hline Total & & 38.200 \\
\hline
\end{tabular}




\section{RESULTADOS}

Na Tabela 1, observa-se que, para os RCBP analisados, os percentuais de casos de câncer pediátrico em relação ao total de casos de câncer variaram de 1,34\% (Goiânia) a 3,85\% (Salvador).

A Tabela 2 mostra as freqüências relativas das leucemias, linfomas, tumores do SNC e outros tumores, em ambos os sexos, observadas nos RCBP do Brasil.

A leucemia destaca-se como neoplasia mais comum, variando entre 15,08\% em Belo Horizonte e 50,00\% em Palmas. Para os linfomas, observou-se que o menor percentual foi em Aracaju (5,26\%), enquanto o maior ocorreu em Natal $(25,84 \%)$. Em relação aos tumores do SNC, o menor percentual foi observado em Natal (5,62\%), enquanto o maior valor foi observado em João Pessoa (22,92\%).

A Figura 1 apresenta as taxas médias de incidência ajustadas por idade, por 100.000 habitantes. As maiores taxas, ajustadas por idade, para leucemias na infância e na adolescência foram encontradas em Natal com 3,19/ 100.000 para o sexo masculino, e em São Paulo com 2,13/100.000 para o sexo feminino. As menores taxas foram observadas em João Pessoa, com 1,11/100.000 e 0,33/100.000, para os sexos masculino e feminino, respectivamente. Para as leucemias, observou-se que a incidência em pacientes do sexo masculino é maior do que nas do sexo feminino em praticamente todas as localidades pesquisadas, exceto em Recife. O comportamento da incidência nas faixas etárias é diferenciado quando são comparados o sexo masculino e o sexo feminino (Figuras 2 e 3). Nota-se que, em geral, ocorre um declínio nas taxas a partir da faixa etária de 5 a 9 anos de idade. As maiores taxas médias específicas de incidência para o sexo masculino (Figura 2) e para o sexo feminino (Figura 3) foram observadas nas faixas etárias de 1 a 4 anos de idade. Conforme detalhado na Tabela 3, quanto ao sexo masculino, em Natal apresentam-se as maiores taxas médias específicas com 19,82/100.000 na faixa etária de 1 a 4 anos e 11,22/ 100.000 na faixa etária de 10 a 14 anos; já no sexo feminino, as maiores taxas médias específicas foram observadas também em Natal, com 10,20/100.000 na faixa etária de 1 a 4 anos, e em Campinas com 5,79/ 100.000 para as crianças menores de 1 ano.

O grupo dos linfomas inclui a doença de Hodgkin e os linfomas não-Hodgkin, sendo estes últimos os mais comuns em crianças e em adolescentes ${ }^{8}$. A Figura 4 apresenta as taxas médias de incidência dos linfomas, ajustadas por idade, observando-se que a incidência mostra um comportamento diferenciado entre os sexos, com maiores taxas em meninos do que em meninas, exceto em Natal, onde se observa o contrário. As maiores

Tabela 1. Freqüência relativa do número de casos novos de tumores pediátricos e não-pediátricos, em ambos os sexos, por RCBP, para o período referido

\begin{tabular}{l|c|c|c|c|c}
\hline \multirow{2}{*}{ RCBP - Período } & \multicolumn{2}{c|}{ Tumores infantis } & \multicolumn{2}{c}{ Tumores não-infantis } & Total (100\%) \\
\cline { 2 - 6 } & casos novos & $\%$ & casos novos & $\%$ & casos novos \\
\hline Salvador (1997-2001) & 532 & 3,85 & 13.296 & 96,15 & 13.828 \\
\hline Manaus (1999) & 70 & 3,71 & 1.817 & 96,29 & 1.887 \\
\hline Belém (1996-1998) & 133 & 3,65 & 3.506 & 96,35 & 3.639 \\
\hline Campinas (1991-1995) & 203 & 3,11 & 6.316 & 96,89 & 6.519 \\
\hline Cuiabá (2000) & 48 & 3,07 & 1.515 & 96,93 & 1.563 \\
\hline João Pessoa (1999-2000) & 48 & 2,98 & 1.563 & 97,02 & 1.611 \\
\hline Distrito Federal (1996-1998) & 424 & 2,95 & 13.930 & 97,05 & 14.354 \\
\hline Recife (1995-1998) & 288 & 2,87 & 9.760 & 97,13 & 10.048 \\
\hline Vitória (1997) & 39 & 2,82 & 1.342 & 97,18 & 1.381 \\
\hline Natal (1998-1999) & 89 & 2,62 & 3.307 & 97,38 & 3.396 \\
\hline Fortaleza (1996) & 83 & 2,52 & 3.206 & 97,48 & 3.289 \\
\hline São Paulo (1997-1998) & 1.675 & 2,15 & 76.391 & 97,85 & 78.066 \\
\hline Aracaju (1996) & 19 & 2,09 & 889 & 97,91 & 908 \\
\hline Palmas (2000) & 2 & 1,69 & 116 & 98,31 & 118 \\
\hline Porto Alegre (1996-1997) & 143 & 1,65 & 8.527 & 98,35 & 8.670 \\
\hline Belo Horizonte (2000) & 179 & 1,54 & 11.440 & 98,46 & 11.619 \\
\hline Goiânia (1996-2000) & 226 & 1,34 & 16.663 & 98,66 & 16.889 \\
\hline
\end{tabular}

Fonte: Registros de Câncer de Base Populacional 
taxas médias de incidência ajustadas por idade foram encontradas em São Paulo, com 1,81/100.000 para o sexo masculino e em Natal, com 2,27/100.000 para o sexo feminino. Já as menores taxas foram observadas em Belém, para ambos os sexos, com 0,48/100.000 para o sexo masculino e $0,35 / 100.000$ para o sexo feminino. Foi observada uma pequena variabilidade nas taxas médias específicas de incidência entre as diversas faixas etárias estudadas no sexo masculino (Figura 5). Já no sexo feminino (Figura 6), observou-se que as taxas

Tabela 2. Freqüência relativa das leucemias, linfomas, tumores do SNC e outros tumores*, em ambos os sexos, por RCBP

\begin{tabular}{|c|c|c|c|c|c|c|c|c|c|}
\hline \multirow[b]{2}{*}{ RCBP - Período } & \multicolumn{2}{|c|}{ Leucemias } & \multicolumn{2}{|c|}{ Linfomas } & \multicolumn{2}{|c|}{$\begin{array}{c}\text { Tumores de } \\
\text { SNC }\end{array}$} & \multicolumn{2}{|c|}{ Outros tumores } & \multirow{2}{*}{$\begin{array}{c}\text { Total } \\
\text { casos } \\
\text { novos } \\
(100 \%)\end{array}$} \\
\hline & $\begin{array}{l}\text { casos } \\
\text { novos }\end{array}$ & $\%$ & $\begin{array}{l}\text { casos } \\
\text { novos }\end{array}$ & $\%$ & $\begin{array}{l}\text { casos } \\
\text { novos }\end{array}$ & $\%$ & $\begin{array}{l}\text { casos } \\
\text { novos }\end{array}$ & $\%$ & \\
\hline Aracaju (1996) & 3 & 15,79 & 1 & 5,26 & 3 & 15,79 & 12 & 63,16 & 19 \\
\hline Belém (1996-1998) & 54 & 40,60 & 20 & 15,04 & 17 & 12,78 & 42 & 31,58 & 133 \\
\hline Belo Horizonte (2000) & 27 & 15,08 & 33 & 18,44 & 31 & 17,32 & 88 & 49,16 & 179 \\
\hline Campinas (1991-1995) & 53 & 26,11 & 27 & 13,30 & 26 & 12,81 & 97 & 47,78 & 203 \\
\hline Cuiabá (2000) & 12 & 25,00 & 5 & 10,42 & 6 & 12,50 & 25 & 52,08 & 48 \\
\hline Distrito Federal (1996-1998) & 104 & 24,53 & 57 & 13,44 & 68 & 16,04 & 195 & 45,99 & 424 \\
\hline Fortaleza (1996) & 15 & 18,07 & 13 & 15,66 & 15 & 18,07 & 40 & 48,19 & 83 \\
\hline Goiânia (1996-2000) & 70 & 30,97 & 48 & 21,24 & 42 & 18,58 & 66 & 29,20 & 226 \\
\hline João Pessoa (1999-2000) & 8 & 16,67 & 7 & 14,58 & 11 & 22,92 & 22 & 45,83 & 48 \\
\hline Manaus (1999) & 32 & 45,71 & 8 & 11,43 & 10 & 14,29 & 20 & 28,57 & 70 \\
\hline Natal (1998-1999) & 31 & 34,83 & 23 & 25,84 & 5 & 5,62 & 30 & 33,71 & 89 \\
\hline Palmas (2000) & 1 & 50,00 & 0 & 0,00 & 0 & 0,00 & 1 & 50,00 & 2 \\
\hline Porto Alegre (1996-1997) & 32 & 22,38 & 28 & 19,58 & 23 & 16,08 & 60 & 41,96 & 143 \\
\hline Recife (1995-1998) & 65 & 22,57 & 52 & 18,06 & 34 & 11,81 & 137 & 47,57 & 288 \\
\hline Salvador (1997-2001) & 112 & 21,05 & 92 & 17,29 & 77 & 14,47 & 251 & 47,18 & 532 \\
\hline São Paulo (1997-1998) & 448 & 26,75 & 249 & 14,87 & 275 & 16,42 & 703 & 41,97 & 1.675 \\
\hline Vitória (1997) & 15 & 38,46 & 7 & 17,95 & 4 & 10,26 & 13 & 33,33 & 39 \\
\hline
\end{tabular}

$\left(^{*}\right)$ Outros tumores incluem todos os grupos de diagnósticos, listados no quadro 1, exceto leucemias, linfomas e tumores do SNC (Grupos IV a XII). Fonte: Registros de Câncer de Base Populacional

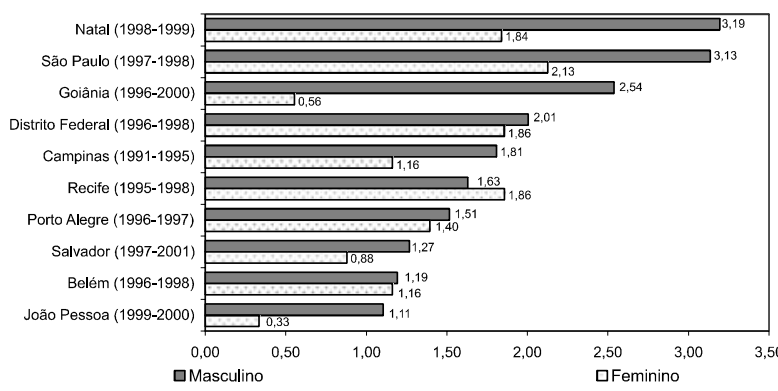

Figura 1. Distribuição das taxas médias de incidência ajustadas por idade*, para leucemias na infância e na adolescência, sexo masculino e feminino, segundo RCBP e período referido

(*) População Padrão Mundial 1960, por 100.000 habitantes Fonte: Registros de Câncer de Base Populacional/IBGE ${ }^{13}$

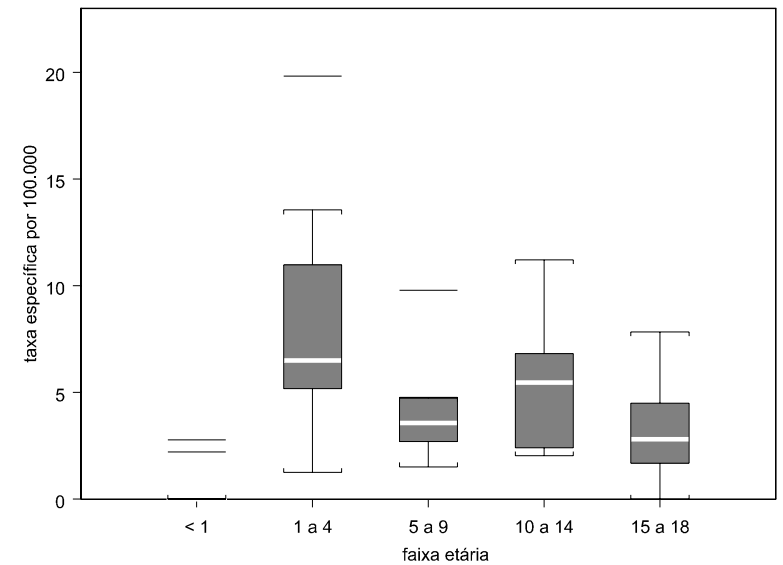

Figura 2. Taxas médias específicas de incidência, por 100.000 habitantes, para leucemias na infância e na adolescência, por faixa etária*, sexo masculino, por RCBP

(*) São Paulo inclui os menores de um ano na faixa etária de 1-4 anos Fonte: Registros de Câncer de Base Populacional//BGE ${ }^{13}$ 
médias específicas de incidência se apresentam ainda mais homogêneas quando comparadas ao sexo masculino. Conforme apresentado na Tabela 3, as maiores taxas médias específicas de incidência foram observadas em Porto Alegre na faixa etária de 15 a 18 anos, com 9,44/100.000, e em São Paulo, também na faixa etária de 15 a 18 anos com 7,96/100.000. Belém apresentou as menores taxas, com $0,55 / 100.000$ na faixa etária de 1 a 4 anos e 1,22/100.000 na faixa etária de 10 a 14 anos. As maiores taxas foram observadas em Natal, em crianças menores de um ano e naquelas entre

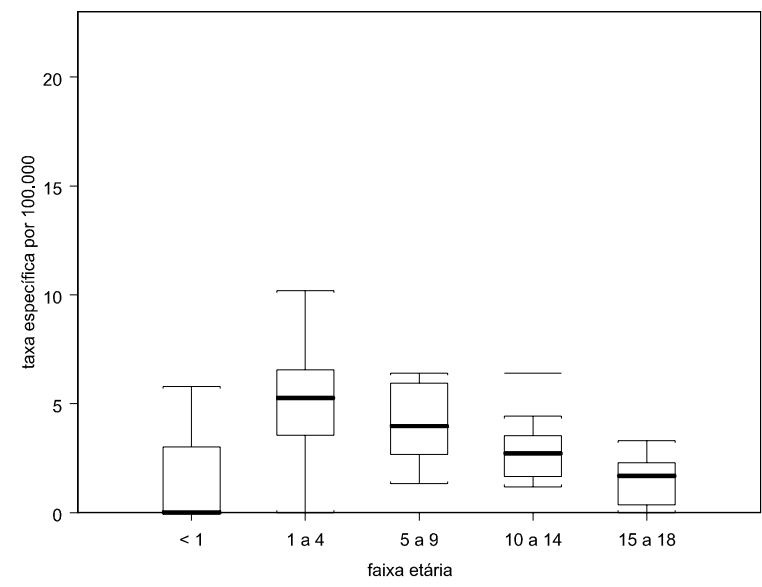

Figura 3. Taxas médias específicas de incidência, por 100.000 habitantes, para leucemias na infância e na adolescência, por faixa etária*, sexo feminino, por RCBP

(*) São Paulo inclui os menores de um ano na faixa etária de 1-4 anos Fonte: Registros de Câncer de Base Populacional/IBGE ${ }^{13}$

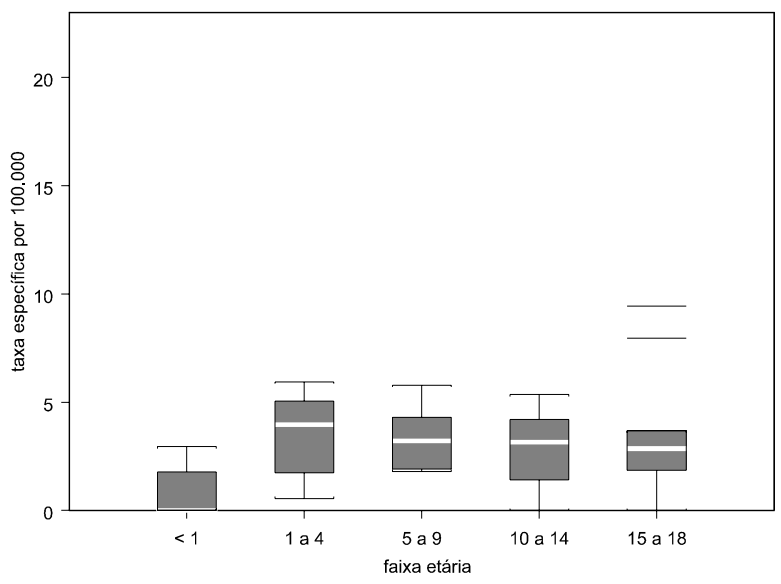

Figura 5. Taxas médias específicas de incidência, por 100.000 habitantes, para linfomas na infância e na adolescência, por faixa etária*, sexo masculino, por RCBP

(*) São Paulo inclui os menores de um ano na faixa etária de 1-4 anos Fonte: Registros de Câncer de Base Populacional//BGE ${ }^{13}$
15 e 18 anos de idade (16,47/100.000 e 8,08/100.000, respectivamente). Belém apresentou a menor taxa na faixa etária de 10 a 14 anos com 0,39/100.000.

A Figura 7 apresenta as taxas médias de incidência ajustadas por idade para os tumores do SNC. Nos RCBP estudados, observou-se predominância no sexo masculino apenas em metade das localidades analisadas. As maiores taxas médias de incidência ajustadas por idade foram encontradas em São Paulo, com 1,67/ 100.000 para o sexo masculino e $1,48 / 100.000$ para o sexo feminino. As menores taxas no sexo masculino

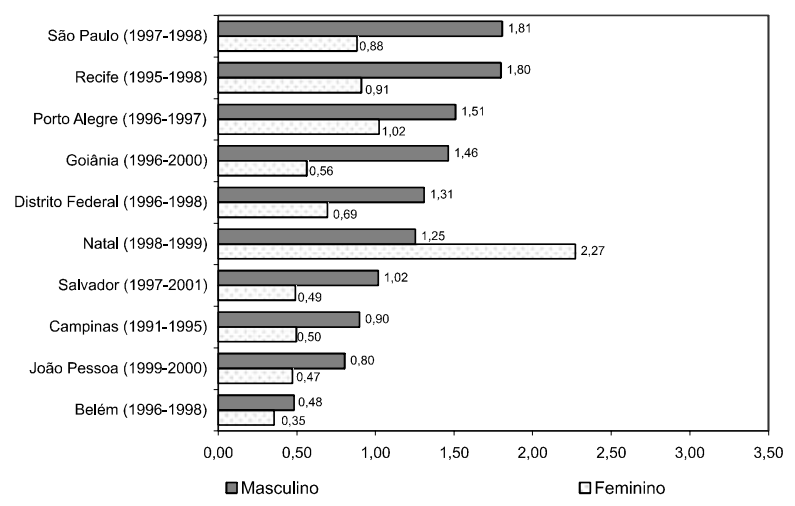

Figura 4. Distribuição das taxas médias de incidência ajustadas* por idade, para linfomas na infância e na adolescência, sexo masculino e feminino, segundo RCBP e período de referência

(*) População Padrão Mundial 1960, por 100.000 habitantes Fonte: Registros de Câncer de Base Populacional/IBGE ${ }^{13}$

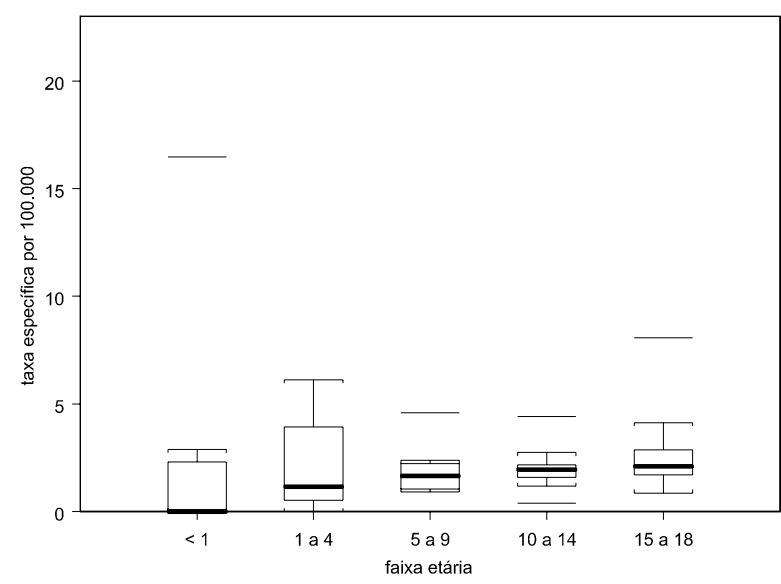

Figura 6. Taxas médias específicas de incidência, por 100.000 habitantes, para linfomas na infância e na adolescência, por faixa etária*, sexo feminino, por RCBP

(*) São Paulo inclui os menores de um ano na faixa etária de 1-4 anos Fonte: Registros de Câncer de Base Populacional/IBGE ${ }^{13}$ 
foram observadas em Natal, com $0,13 / 100.000$, e no sexo feminino em Belém, com 0,44/100.000. Para o sexo masculino (Figura 8), nas localidades analisadas, a faixa etária que apresentou maiores valores para incidência foi a de 1 a 4 anos. Para o sexo feminino (Figura 9), as maiores taxas médias específicas de incidência também foram observadas na faixa etária de 1 a 4 anos. Com base nas informações apresentadas na
Tabela 3, em Porto Alegre observou-se a maior taxa média específica de incidência, 6,32/100.000 para a faixa etária de 1 a 4 anos. Já a menor taxa foi observada em Belém, com 0,55/100.000, para a faixa etária de $1 \mathrm{a}$ 4 anos. A maior taxa específica de incidência foi de 5,83/100.000 em Recife para a faixa etária de 1 a 4 anos. A menor taxa, 0,41/100.000, foi encontrada em Salvador, para a faixa etária de 5 a 9 anos.

Tabela 3. Taxas médias específicas de incidência, por 100.000 habitantes, para leucemias, linfomas e tumores do SNC na infância e na adolescência, por faixa etária *, ambos os sexos, por RCBP

\begin{tabular}{|c|c|c|c|c|c|c|c|c|c|c|c|c|c|c|c|c|}
\hline \multirow{3}{*}{ Sexo } & \multirow{3}{*}{ RCBP - (Período) } & \multicolumn{5}{|c|}{ Leucemias } & \multicolumn{5}{|c|}{ Linfomas } & \multicolumn{5}{|c|}{ Tumores de SNC } \\
\hline & & \multicolumn{5}{|c|}{ Faixa etária } & \multicolumn{5}{|c|}{ Faixa etária } & \multicolumn{5}{|c|}{ Faixa etária } \\
\hline & & $<1$ & $1-4$ & 5-9 & $10-14$ & $15-18$ & $<1$ & $1-4$ & $5-9$ & $10-14$ & $15-18$ & $<1$ & $1-4$ & $5-9$ & 10.14 & $15-18$ \\
\hline \multirow{10}{*}{$\begin{array}{l}\stackrel{\text { 을 }}{\underline{z}} \\
\text { 믈 }\end{array}$} & Belém (1996-1998) & 0,00 & 5,52 & 2,71 & 2,03 & 2,91 & 0,00 & 0,55 & 1,80 & 1,22 & 1,94 & 0,00 & 0,55 & 0,00 & 0,81 & 2,42 \\
\hline & Campinas (1991-1995) & 2,78 & 10,98 & 3,54 & 2,34 & 1,69 & 2,78 & 3,43 & 2,53 & 1,41 & 1,69 & 2,78 & 1,37 & 3,04 & 2,81 & 1,69 \\
\hline & $\begin{array}{c}\text { Distrito Federal } \\
\text { (1996-1998) }\end{array}$ & 0,00 & 7,32 & 2,98 & 7,56 & 4,50 & 1,78 & 3,66 & 3,72 & 3,09 & 3,68 & 3,55 & 3,20 & 1,86 & 4,13 & 2,86 \\
\hline & Goiânia (1996-2000) & 2,22 & 13,55 & 3,86 & 4,79 & 5,09 & 0,00 & 5,65 & 4,29 & 3,99 & 1,85 & 0,00 & 1,69 & 3,86 & 1,20 & 2,31 \\
\hline & $\begin{array}{l}\text { João Pessoa } \\
(1999-2000)\end{array}$ & 0,00 & 4,88 & 1,82 & 5,05 & 0,00 & 0,00 & 4,88 & 1,82 & 0,00 & 2,13 & 0,00 & 4,88 & 1,82 & 0,00 & 4,27 \\
\hline & Natal (1998-1999) & 0,00 & 19,82 & 1,52 & 11,22 & 1,80 & 0,00 & 5,95 & 3,04 & 4,21 & 0,00 & 0,00 & 0,00 & 0,00 & 1,40 & 0,00 \\
\hline & $\begin{array}{l}\text { Porto Alegre } \\
(1996-1997)\end{array}$ & 0,00 & 1,26 & 4,78 & 6,82 & 4,19 & 0,00 & 5,06 & 1,91 & 1,70 & 9,44 & 0,00 & 6,32 & 2,87 & 2,56 & 2,10 \\
\hline & Recife (1995-1998) & 0,00 & 5,66 & 4,74 & 5,85 & 1,19 & 2,96 & 4,25 & 5,79 & 5,36 & 3,58 & 0,00 & 4,25 & 2,11 & 2,92 & 1,19 \\
\hline & Salvador (1997-2001) & 0,00 & 5,17 & 3,59 & 2,41 & 2,71 & 0,00 & 1,29 & 3,39 & 3,21 & 3,68 & 2,17 & 3,10 & 1,00 & 1,77 & 2,13 \\
\hline & São Paulo (1997-1998)* & - & 8,55 & 9,79 & 6,29 & 7,83 & - & 1,74 & 5,66 & 5,10 & 7,96 & - & 3,84 & 4,95 & 3,90 & 4,99 \\
\hline \multirow{10}{*}{ 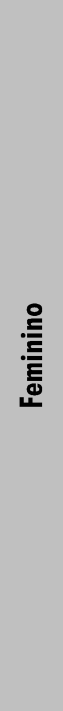 } & Belém (1996-1998) & 0,00 & 4,62 & 3,22 & 2,71 & 2,14 & 2,31 & 1,15 & 0,92 & 0,39 & 0,86 & 0,00 & 2,89 & 0,92 & 0,78 & 0,00 \\
\hline & Campinas (1991-1995) & 5,79 & 3,55 & 4,72 & 1,44 & 1,13 & 2,89 & 0,71 & 1,05 & 1,92 & 1,13 & 0,00 & 3,55 & 0,00 & 1,44 & 0,00 \\
\hline & $\begin{array}{c}\text { Distrito Federal } \\
\text { (1996-1998) }\end{array}$ & 3,65 & 8,45 & 4,76 & 2,71 & 3,30 & 1,82 & 0,47 & 2,38 & 2,03 & 2,57 & 3,65 & 5,17 & 3,81 & 2,37 & 2,57 \\
\hline & Goiânia (1996-2000) & 0,00 & 2,32 & 1,33 & 1,18 & 1,28 & 0,00 & 1,16 & 2,22 & 1,18 & 1,71 & 2,29 & 3,49 & 3,56 & 1,97 & 0,85 \\
\hline & $\begin{array}{l}\text { João Pessoa } \\
(1999-2000)\end{array}$ & 0,00 & 0,00 & 1,86 & 1,66 & 0,00 & 0,00 & 0,00 & 1,86 & 1,66 & 1,90 & 0,00 & 0,00 & 5,57 & 1,66 & 3,79 \\
\hline & Natal (1998-1999) & 0,00 & 10,20 & 6,13 & 2,75 & 0,00 & 16,47 & 6,12 & 4,60 & 2,75 & 8,08 & 0,00 & 0,00 & 1,53 & 1,37 & 3,23 \\
\hline & $\begin{array}{l}\text { Porto Alegre } \\
(1996-1997)\end{array}$ & 0,00 & 6,56 & 2,99 & 3,53 & 2,08 & 0,00 & 3,93 & 1,00 & 4,41 & 2,08 & 0,00 & 2,62 & 2,99 & 2,65 & 2,08 \\
\hline & Recife (1995-1998) & 3,01 & 6,56 & 5,94 & 4,42 & 2,29 & 0,00 & 4,37 & 1,08 & 1,96 & 2,86 & 0,00 & 5,83 & 1,08 & 1,47 & 1,72 \\
\hline & Salvador (1997-2001) & 0,00 & 3,72 & 2,67 & 2,55 & 0,35 & 0,00 & 0,53 & 1,44 & 1,59 & 2,11 & 0,00 & 4,52 & 0,41 & 2,07 & 0,70 \\
\hline & São Paulo (1997-1998)* & - & 5,89 & 6,40 & 6,40 & 2,84 & - & 1,54 & 2,05 & 2,17 & 4,13 & - & 3,20 & 4,83 & 3,80 & 3,74 \\
\hline
\end{tabular}

(*) São Paulo inclui os menores de um ano na faixa etária de 1-4 anos

Fonte: Registros de Câncer de Base Populacional/IBGE ${ }^{13}$ 


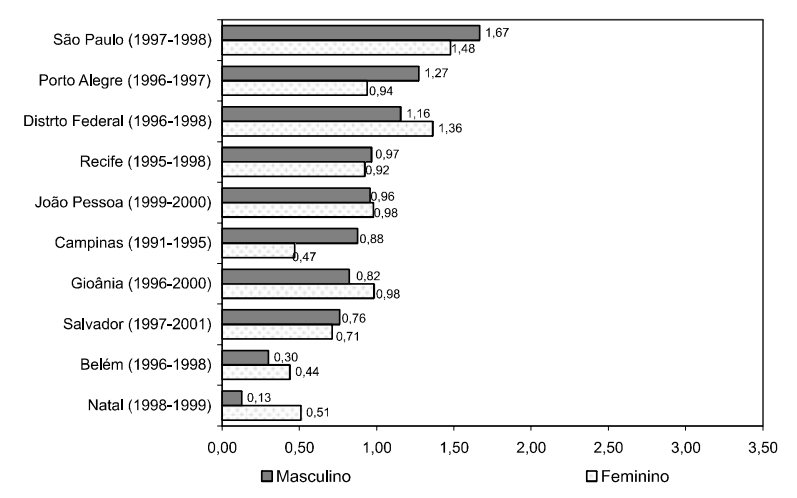

Figura 7. Distribuição das taxas médias de incidência ajustadas* por idade, para tumores do SNC na infância e na adolescência, sexo masculino e feminino, segundo RCBP e período de referência

(*) População Padrão Mundial 1960, por 100.000 habitantes

Fonte: Registros de Câncer de Base Populacional/[BGE ${ }^{13}$

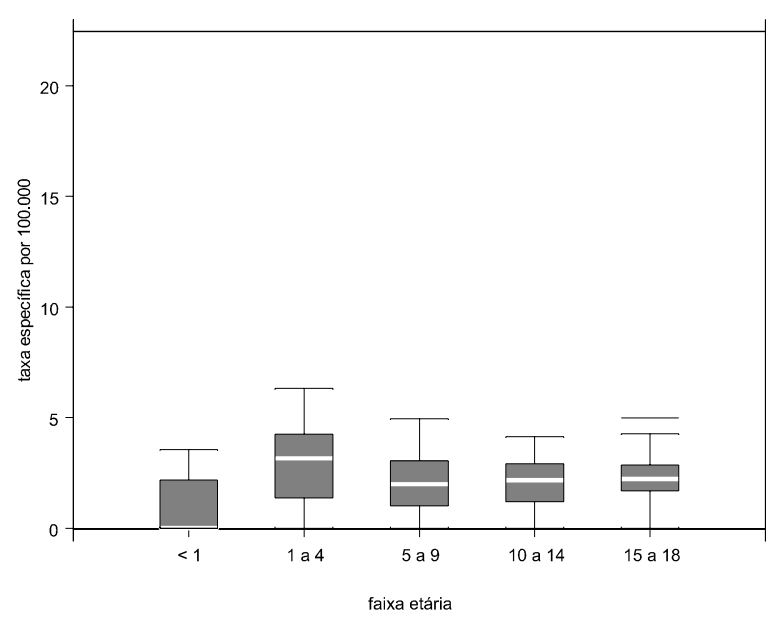

Figura 8. Taxas médias específicas de incidência, por 100.000, para tumores do SNC na infância e na adolescência, por faixa etária*, sexo masculino, por RCBP

$\left(^{*}\right)$ São Paulo inclui os menores de um ano na faixa etária de 1-4 anos Fonte: Registros de Câncer de Base Populacional//BGE ${ }^{13}$

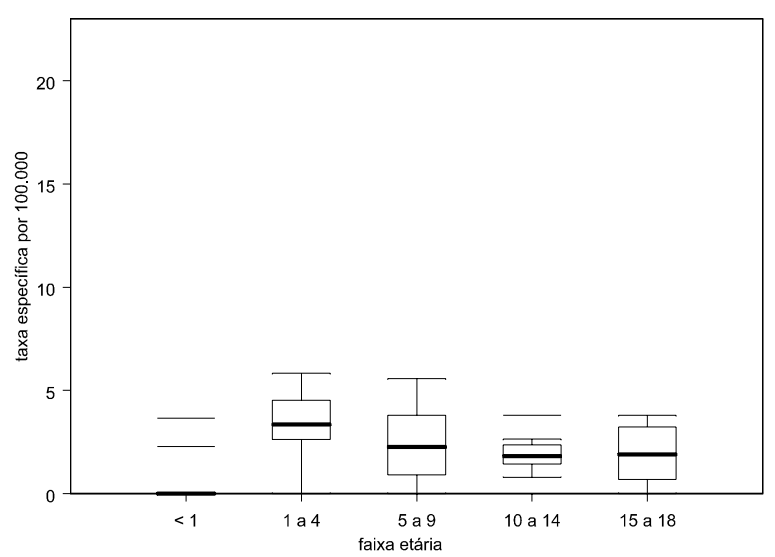

Figura 9. Taxas médias específicas de incidência, por 100.000 habitantes, para tumores do SNC na infância e na adolescência, por faixa etária*, sexo feminino, por RCBP

(*) São Paulo inclui os menores de um ano na faixa etária de 1-4 anos Fonte: Registros de Câncer de Base Populacional//BGE ${ }^{13}$

\section{DISCUSSÃO}

Em geral, os valores observados para a freqüência relativa de câncer na infância estiveram dentro dos padrōes internacionais ${ }^{9}$; entretanto, os dados de Salvador e de Manaus foram superiores àqueles referidos pela literatura, 3,85\% e 3,71\%, respectivamente. Uma possível explicação para os números de Salvador pode ser o fato de existir um centro de referência para tumores pediátricos na cidade, o que induz a migração de casos do interior do estado. Devido ao longo tempo de tratamento, muitas vezes os pacientes alojam-se na capital, passando a figurar como caso incidente desta. Já para Manaus, além desta provável explicação, podem ter sido computados casos prevalentes, uma vez que se trata do primeiro ano de informações consolidadas deste RCBP.

Para as leucemias, os percentuais observados nos 17 RCBP analisados são análogos aos encontrados na literatura científica ${ }^{9}$, variando de $25 \%$ a $35 \%$ de todas as neoplasias malignas pediátricas ${ }^{1}$. Na maioria dos países, crianças menores de cinco anos são as mais freqüentemente acometidas por este tipo de neoplasia ${ }^{6}$. Corroborando estes achados, observou-se, neste trabalho, sua maior incidência em crianças de 1 a 4 anos. Internacionalmente, as taxas médias de incidência ajustadas por idade, por 100.000 habitantes, para a faixa etária de 0 a 19 anos, variaram entre 2,54/100.000 (Islândia, 1993-1997) e 5,62/100.000 (Vêneto/Itália, 1993-1996), para ambos os sexos9. Já para os RCBP brasileiros analisados, as taxas médias de incidência, ajustadas por idade, variaram de 0,33/100.000 (João Pessoa) a 3,19/100.000 (Natal), no sexo masculino. A faixa etária com maior taxa média específica de incidência observada internacionalmente foi a de 0 a 4 anos, com valores que chegam a 11,18/100.000 (Piemonte/Itália, 1993-1997)9. Nos RCBP brasileiros, a maior taxa média específica de incidência observada foi 19,82/100.000, no sexo masculino, na faixa etária de 1 a 4 anos (em Natal).

Nos países desenvolvidos, sabe-se que os linfomas abrangem cerca de $7 \%$ a $18 \%$ das neoplasias pediátricas, ocupando o terceiro lugar. Já nos países em vias de desenvolvimento, correspondem ao segundo lugar, ficando atrás apenas das leucemias ${ }^{10}$. A maioria das localidades incluída neste estudo apresentou percentuais semelhantes aos referidos para países em desenvolvimento. Uma das maiores taxas médias de incidência ajustadas por idade foi encontrada na cidade de Toscana/Itália $(5,08 / 100.000)^{9}$. Já a menor taxa foi encontrada na Irlanda do Norte (1993-1996), com 1,59/ 100.0009. Para o Brasil, dos RCBP analisados, a maior 
taxa média de incidência ajustada por idade foi de 2,27/ 100.000, em Natal, no sexo feminino. Com relação às taxas médias específicas de incidência por idade, a maior taxa observada, 10,46/100.000, foi encontrada na Islândia (1993-1997), na faixa etária de 15 a 19 anos? Neste estudo, observou-se que as maiores taxas médias específicas de incidência foram observadas em Natal, em crianças do sexo feminino, menores de um ano $(16,47 / 100.000)$, não havendo uma faixa etária específica mais acometida por este grupo de neoplasias, o que pode ser um reflexo da sua variação morfológica.

No que diz respeito aos tumores do SNC, estima-se que cerca de $8 \%$ a $15 \%$ das neoplasias pediátricas são representadas por este grupo ${ }^{6}$, sendo o mais freqüente grupo de neoplasias sólidas malignas na faixa pediátrica ${ }^{11}$. Em geral, esse tipo de neoplasia acomete mais meninos do que meninas ${ }^{8}$. Em países desenvolvidos, os tumores do SNC representam o segundo grupo de diagnóstico mais comum, contribuindo com cerca de $17 \%$ a $25 \%$ das neoplasias na infância. Para os países em vias de desenvolvimento, constituem o terceiro tipo de neoplasia mais incidente ${ }^{1}$. No Brasil, uma observação interessante é que as informações obtidas pelos RCBP não reproduzem as encontradas na literatura mundial, que referem o sexo masculino como apresentando taxas de incidência maiores do que as do sexo feminino ${ }^{8}$. Além disso, as maiores taxas médias específicas de incidência por idade foram encontradas na faixa etária de 1 a 4 anos, o que reflete um comportamento diferente do encontrado por outros autores, que referem um pico de incidência entre as idades de 5 a 10 anos $^{8}$. Em cinco localidades, o sexo masculino apresentou as maiores taxas de incidência, enquanto nas outras cinco os maiores valores foram encontrados no sexo feminino. Internacionalmente, o registro da Dinamarca (19931997) apresentou a maior taxa média de incidência ajustada por idade, 4,82/100.000. Já a menor taxa foi encontrada no registro da cidade de Doubs/França (1993-1996), 0,69/100.0009. Para os RCBP brasileiros analisados, o maior valor observado foi $1,67 / 100.000$, em São Paulo, no sexo masculino. A maior taxa média específica de incidência observada no mundo foi encontrada na Islândia (1993-1997), 6,63/100.000, para a faixa etária de 10 a 14 anos9. Em relação ao Brasil, as maiores incidências foram observadas em Porto Alegre, na faixa etária de 1 a 4 anos, no sexo masculino, com 6,32/100.000.

Ressalta-se que na maior parte dos RCBP analisados, há um percentual considerável de tumores mal classificados (Grupo XII), que varia de 0\% (Palmas) a 56,7\% (Cuiabá). Tal fato pode também contribuir para a diferença no perfil de incidência do câncer pediátrico observado no país, sobretudo para os tumores do SNC. Entretanto, de uma maneira geral, as informaçōes sobre tumores pediátricos, obtidas a partir dos 17 RCBP do Brasil, são compatíveis com aquelas referenciadas pela literatura científica para países em desenvolvimento.

Recentemente, o RCBP do município de São Paulo publicou uma análise da incidência, da mortalidade e da sobrevida do câncer da infância para a sua área de cobertura $^{12}$.

Espera-se, com este trabalho que pretendeu descrever o perfil da incidência dos tumores pediátricos no Brasil, contribuir para divulgar e ampliar as informações disponíveis, apoiar os gestores de saúde na tomada de decisão para o enfrentamento do câncer pediátrico, bem como fornecer subsídios para a formulação de hipóteses a serem investigadas futuramente. A análise destas informações deve sempre levar em consideração as migrações interestaduais, a concentração de centros de tratamentos especializados nas capitais, bem como a oferta de leitos específicos para o tratamento do câncer na infância e na adolescência. Recomenda-se, além disso, cautela na interpretação das informaçōes, sobretudo, para os locais onde há apenas um ano de informação disponível.

\section{REFERÊNCIAS}

1. Parkin DM, Stiller CA, Draper GJ, Bieber CA, Terracini B, Young JL, editors. International incidence of childhood cancer. Lyon (France): International Agency for Research on Cancer; 1988 (IARC. Scientific Publications n ${ }^{\circ} 87$ ).

2. Kramárová E, Stiller CA, Ferlay J, Parkin DM, Draper GJ, Michaelis J, Neglia J, Qureshi S. Classificação internacional do câncer na infância 1996. IARC - Relatório técnico N 29. Rio de Janeiro: INCA; 1999.

3. Malkin D. Cancer of childhood. In: DeVita VT Jr, Hellman $S$, Rosenberg SA, editors. Cancer: principles and practice of oncology. 5th ed. New York: Lippincott-Raven; 1997:2083-2091.

4. Jensen OM, Parkin DM, MacLennan R, Muir CS, Skeet RG, organizadores. Registro de câncer: princípios e métodos. IARC - Publicação Científica no ${ }^{9}$ 5. Rio de Janeiro: INCA; 1995.

5. SisBasepop: Sistema de Registro de Câncer de Base Populacional [programa de computador] versão 5.3. Rio de Janeiro: INCA; 2003.

6. Little J. Epidemiology of childhood cancer. Lyon (France): International Agency for Research on Cancer; $n^{\circ} 149 ; 1999$.

7. Parkin DM, Kramárová E. Materials and methods. In: Parkin DM, Kramárová E, Draper GJ, Masuyer E, Michaelis J, Neglia J, et al. International incidence of childhood cancer. Lyon (France): International Agency for Research on Cancer; 1998:3-14. 
8. National Childhood Cancer Foundation. What is childhood cancer? [cited 2003 Sept 9]. Available from:<http://www.childhoodcancerawareness.org/ ccancer.asp $>$

9. Automated Childhood Cancer Information System. [cited 2003 Oct 3]. Available from: <http://www-dep.iarc.fr/accis/ data.htm>

10. Braga PE, Latorre MRDO, Curado MP. Câncer na infância: análise comparativa da incidência, mortalidade e sobrevida em Goiânia (Brasil) e outros países. Cad. Saúde Pública. 2002;18(1):33-44.

11. Furrer AA, Osório CAM, Sanematsu P, Ferrigno R. Tumores de sistema nervoso central: revisão para o pediatra geral. São Paulo: Centro de Tratamento e Pesquisa do Hospital do Câncer A.C.Camargo. [acesso em out 2003]. Disponível em: <http://www.hcanc.org.br/outrasinfs/ensaios/ sncped 1.html>

12. Mirra AP, Latorre MRDO, Veneziano DB. Incidência, mortalidade e sobrevida do câncer na infância no município de São Paulo. São Paulo: Registro de Câncer de São Paulo; 2004.

13. Instituto Brasileiro de Geografia e Estatística. SIDRA Banco de Dados Agregados: população residente-contagem populacional 1996: municípios. [acesso em out 2003]. Disponível em; http://www.sidra.ibge.gov.br/bda

\section{Abstract}

Despite growing interest in the epidemiology of childhood cancers, but few studies have been done on pediatric tumors in Brazil. To encourage etiological studies, the current study presents data on the incidence of the main childhood cancers from 17 of 22 Brazilian population-based cancer registries (PBCR) for which such information is available. An information system called SisBasepop, developed by the National Cancer Institute (INCA), was used to collect and analyze data from the registries. Leukemia was the most frequent childhood cancer, comprising $15 \%-45 \%$ of all malignancies. Lymphoma was the second most common malignancy in children (5\%-25\%), followed by central nervous system tumors (5\%-22\%). We conclude that SisBasepop is a viable system to provide population-based incidence data obtained through PBCR.

Key words: Neoplasm; Incidence; Children; Adolescents; Brazil. 